\title{
Production Potential Appraisal: A Case Study in Jammalamadugu- Proddutur Tract of Pennar River Basin in YSR Kadapa District, Andhra Pradesh, India
}

\author{
D. V. Sujatha ${ }^{1}$, M. V. S. Naidu ${ }^{*}$, B. P. Bhaskar ${ }^{1}$, \\ D. Subramanyam ${ }^{2}$ and B. Ravindra Reddy ${ }^{3}$
}

${ }^{1}$ Department of Soil Science and Agricultural Chemistry, ${ }^{2}$ Department of Agronomy, ${ }^{3}$ Department of Statistics, S.V. Agricultural College, Acharya N.G. Ranga Agricultural University, Tirupati, Andhra Pradesh, India

*Corresponding author

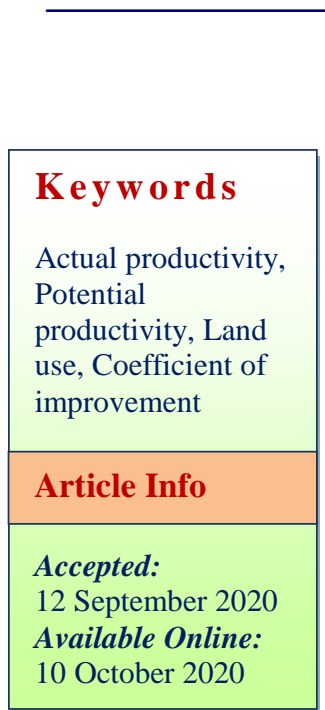

\section{A B S T R A C T}

Eleven typical pedons representing major landforms in semiarid ecosystems of Jammalamadugu - Proddutur tract of Pennar river basin in YSR Kadapa district viz., summits, backslopes and foot slopes developed from lower Gandikota formation with Quartzite (conglomerate, quartzite and rippled siltstone) and upper Tadipatri formation with purple shale, (siltstone and carbonate complex) under varying land uses were evaluated for actual and potential productivity. The actual productivity of P1 (21.54) and P2 (20.41) situated on summits was average and the potential productivity was good. Similarly, the actual productivity of P3 to P11 located on back slopes and foot slopes was poor and the potential productivity was good. However, actual and potential productivity are increased from summits to foot slopes. The coefficient of improvement ranged from (Ci) from 1.78 (Pedons1 and2) to 4.57 (Pedons 3, 7, 8, 10 and 11). The study revealed that, implementation of judicious soil management practices such as application of green manure/ FYM/crop residues/, contour farming and contour bunding and mixing of soil with tank silt helps not only to achieve sustainable yields but also sustains the soil productivity.

\section{Introduction}

Soil is a critical natural resource and maintaining soils in the state of high productivity on sustainable basis needs periodical evaluation. The knowledge of soils with respect to their extent, distribution, characteristics and potential use is important for optimizing land use. Nature provides soil and climate for farming but fertility of soil differs from place to place for which availability of nutritional elements is responsible. Soil fertility specialists need well characterized sites with similar soil and other ecological conditions in order to carry out meaningful fertilizer trails.

Indiscriminate use of finite soil resources coupled with lack of proper management has led to degradation echoing the concern of 
planners, researchers and farmers (Sharma, 2006). Management of finite soil resources in agro -ecological regions is highly essential for not only to increase the yields of different crops but also to sustain soil productivity. Jammalamadugu - Proddutur tract of Pennar river basin in YSR Kadapa district falls under semiarid agro- ecological region having variety of soils. Evaluation of soils for different land uses is the first milestone to develop sustainable and eco-friendly land use models. Though sporadic information is available for the soils of Pulivendula region in Kadapa district (Niranjana et al., 2011), but no information is available especially on soil potential productivity pertaining to Jammalamadugu - Proddutur tract of Kadapa district in Andhra Pradesh. Evaluation of soils for potential productivity plays a pivotal role not only to sustain soil fertility but alsoto improve soil productivity. Hence, present study was taken up to study the Jammalamadugu - Proddutur of tract of Pennar river basin in YSR Kadapa district in Andhra Pradesh.

\section{Materials and Methods}

The study area liesin between $14^{\circ} 45^{\prime}$ and $14^{\circ} 53^{\prime}$ North latitudes and $78^{\circ} 21^{\prime}$ to $78^{\circ} 36^{\prime}$ East longitudes (Fig. 1 and 2) at an elevation of ranging from $160-330 \mathrm{~m}$ (msl) The area qualified for semi - arid monsoonic climate with a distinct summer, winter and rainy seasons with a mean annual rainfall of 943 $\mathrm{mm}$ (2011-2020). The mean annual temperature is $25.9^{\circ} \mathrm{Cwith}$ a mean annual summer temperature of $28.01^{\circ} \mathrm{C}$ and mean annual winter temperature of $23.3^{\circ} \mathrm{C}$. The maximum temperature for the last 10 years is $42.6^{\circ} \mathrm{C}$, recorded in the month of May whereas the minimum temperature is $12^{\circ} \mathrm{C}$, noticed in the month of December. The study area qualifies for ustic soil moisture regime and as iso-hyperthermic soil temperature regime. The natural vegetation in the study area comprises of Azadirachta indica,
Parthenium hysterophorus, Cynodon dactylon, Calotropis gigantia, Acacia nilotica, Tephrosia perpurea, Amaranthus viridis, Cypurus rotundus, Pongamia pinneta, etc.

\section{Field survey}

A reconnaissance soil survey was conducted in Jammalamadugu - Proddutur tract of Pennar river basin in YSR Kadapa district using toposheet of 1:50,000 scale as per the procedure outlined by AIS \& LUS (1970). After traversing the area, based on the visual observations and based on the variations in soil site characteristics (Table 1), twenty sites were selected by following the procedure outlined by soil survey division staff (2000). Soil correlation exercise yielded eleven typical pedons i.e., four pedons in summits, four pedons in back slopes and three in foots slopes in the study area. Horizon-wise soil samples were collected forlaboratory analysis. Processed soil samples $(<2 \mathrm{~mm})$ were analyzed for various physic- chemical properties such as organic carbon, texture and cation exchange capacity by adopting standard analytical procedures (Bower et al., 1952; Jackson 1973; Piper, 1966). The soils were classified up to the sub-groups levels by following USDA soil taxonomy (Soil Survey Staff 2014).

The Riquier's parametric approach (1970) suggests nine factors for the calculation of productivity index, viz., $\mathrm{P}-$ productivity index, $\mathrm{H}$ - soil moisture content, D - drainage, $\mathrm{P}$-effective soil depth, T - texture/structure, N - base saturation, $\mathrm{S}$ - soluble salts, $\mathrm{O}$ - organic matter, $\mathrm{A}-$ mineral exchange capacity and $\mathrm{M}$ - mineral reserves.

$\mathrm{P}=\mathrm{H}$ X D X P X T X N/S X O X A X M (1)

Each factor is rated on a scale from 0 to 100 , the actual percentage being multiplied by each other. The resultant index of productivity 
also lying between 0 and 100 is set against a scale placing the soil in one or other of five productivity classes. After employing all the possible improvement factors, the potential productivity rating was worked out by assigning different grades. The coefficient of improvement $(\mathrm{Ci})$ was worked out based on the actual productivity and potential productivity ratings.

Coefficient of improvement $(\mathrm{Ci})=$ Potential productivity rating/Actual productivity rating

\section{Results and Discussion}

\section{Actual and potential productivity}

In the present study, actual and potential productivity of soils / pedons are determined by following parametric approach of Riquier et al (1970), who suggested that productivity index for evaluating soils for the commonly growing crops of the area. Accordingly, the productivity index for soils under the study was calculated by considering nine factors which were related to soil productivity.

Table.1 Landscape characteristics of Pedons

\begin{tabular}{|c|c|c|c|c|c|}
\hline $\begin{array}{l}\text { Pedons / } \\
\text { Villages }\end{array}$ & Location & $\begin{array}{l}\text { Elevation } \\
\text { above } \\
\text { MSL (m) }\end{array}$ & $\begin{array}{l}\text { Physio- } \\
\text { graphy }\end{array}$ & $\begin{array}{c}\text { Slope } \\
(\%)\end{array}$ & Drainage \\
\hline P1 Kottala & $\begin{array}{l}\text { N } 14^{0} 45 \text { '55.1" } \\
\text { E } 078^{0} 21 ' 51.6^{\prime \prime}\end{array}$ & 330 & Summits & $5-10$ & $\begin{array}{c}\text { Well } \\
\text { drained }\end{array}$ \\
\hline P2Mallambhavi & $\begin{array}{l}\mathrm{N} 14^{\circ} 46^{\prime} 53.1^{\prime \prime} \\
\text { E } 078^{0} 22^{\prime} 41.1^{\prime \prime}\end{array}$ & 260 & Summits & $5-10$ & $\begin{array}{c}\text { Well } \\
\text { drained }\end{array}$ \\
\hline P3 Kondapuram & $\begin{array}{l}\mathrm{N} 14^{0} 48^{\prime} 47.1^{\prime \prime} \\
\text { E } 078^{0} 22^{\prime} 38.8^{\prime \prime}\end{array}$ & 200 & Backslope & $3-5$ & $\begin{array}{c}\text { Somewhat } \\
\text { poorly drained }\end{array}$ \\
\hline P4 Kanneluru & $\begin{array}{l}\mathrm{N} 14^{0} 50^{\prime} 37.8^{\prime \prime} \\
\mathrm{E} 078^{0} 24^{\prime} 29.9^{\prime \prime}\end{array}$ & 188 & Backslope & $1-3$ & $\begin{array}{c}\text { Somewhat } \\
\text { poorly drained }\end{array}$ \\
\hline P5 Seshareddipalli & $\begin{array}{l}\mathrm{N} 14^{\mathrm{O}} 5030.1^{\prime \prime} \\
\mathrm{E} 078^{\mathrm{O}} 3625.1^{\prime \prime}\end{array}$ & 183 & Footslope & $3-5$ & $\begin{array}{c}\text { Somewhat } \\
\text { poorly drained }\end{array}$ \\
\hline P6 Peddapasupula & $\begin{array}{l}\text { N } 14^{0} 53^{\prime} 07.9^{\prime \prime} \\
\text { E } 078^{\circ} 26^{\prime} 50.6^{\prime \prime}\end{array}$ & 174 & Footslope & $1-3$ & $\begin{array}{c}\text { Somewhat } \\
\text { poorly drained }\end{array}$ \\
\hline P7 Sumanchipalli & $\begin{array}{c}\text { N } 14^{0} 53^{\prime} 07.9 \\
\text { E } 078^{0} 26^{\prime} 50.6^{\prime \prime}\end{array}$ & 230 & Summits & $0-1$ & $\begin{array}{c}\text { Somewhat } \\
\text { poorly drained }\end{array}$ \\
\hline P8Chinnadandluru & $\begin{array}{l}\mathrm{N} 14^{0} 46^{\prime} 26.3^{\prime \prime} \\
\text { E } 078^{0} 28^{\prime} 52.1^{\prime \prime}\end{array}$ & 200 & Summits & $0-1$ & $\begin{array}{c}\text { Somewhat } \\
\text { poorly drained }\end{array}$ \\
\hline P9 Chouduru & $\begin{array}{l}\mathrm{N} 14^{0} 46^{\prime} 55.5^{\prime \prime} \\
\text { E } 078^{0} 32^{\prime} 48.4^{\prime \prime}\end{array}$ & 180 & Backslope & $1-3$ & $\begin{array}{c}\text { Somewhat } \\
\text { poorly drained }\end{array}$ \\
\hline P10 Kakirenipalle & $\begin{array}{l}\mathrm{N} 14^{0} 47^{\prime} 16.6 \\
\mathrm{E} 078^{0} 28^{\prime} 51.0^{\prime \prime}\end{array}$ & 175 & Backslope & $1-3$ & $\begin{array}{c}\text { Somewhat } \\
\text { poorly drained }\end{array}$ \\
\hline P11 Pottipadu & $\begin{array}{l}\text { N } 14^{0} 49^{\prime} 17.5^{\prime \prime} \\
\text { E } 078^{0} 32 \text { ' } 12.7^{\prime \prime}\end{array}$ & 160 & Footslope & $1-3$ & $\begin{array}{c}\text { Somewhat } \\
\text { poorly drained }\end{array}$ \\
\hline
\end{tabular}


Table.2 Actual productivity index and Actual productivity class of Jammalamadugu -Proddutur tract of Pennar river basin in YSRKadapa district

\begin{tabular}{|c|c|c|c|c|c|c|c|c|c|c|c|}
\hline $\begin{array}{l}\text { Pedon } \\
\text { No }\end{array}$ & $\begin{array}{l}\text { Moisture } \\
\text { (H) }\end{array}$ & $\begin{array}{c}\text { Drainage } \\
\text { (D) }\end{array}$ & $\begin{array}{l}\text { Effective } \\
\text { depth } \\
\text { (P) }\end{array}$ & $\begin{array}{c}\text { Texture/ } \\
\text { Structure } \\
\text { (T) }\end{array}$ & $\begin{array}{c}\text { Base } \\
\text { saturation } \\
\text { (N) }\end{array}$ & $\begin{array}{c}\text { Soluble } \\
\text { salts } \\
\text { (S) }\end{array}$ & $\begin{array}{c}\text { Organic } \\
\text { matter } \\
\text { content }(\mathbf{O})\end{array}$ & $\begin{array}{c}\text { Mineral } \\
\text { exchange } \\
\text { capacity (A) }\end{array}$ & $\begin{array}{c}\text { Mineral } \\
\text { reserve } \\
\text { (M) }\end{array}$ & $\begin{array}{c}\text { Actual } \\
\text { productivity } \\
\text { index }\end{array}$ & $\begin{array}{c}\text { Actual } \\
\text { productivity } \\
\text { class }\end{array}$ \\
\hline 1 & $\mathrm{H} 4 \mathrm{~b}(90)$ & D4(100) & P3(50) & T6b(90) & N5(100) & S3(80) & O1(70) & A2(95) & $\mathrm{M} 2 \mathrm{c}(100)$ & 21.54 & Average \\
\hline 2 & $\mathrm{H} 4 \mathrm{~b}(90)$ & D4(100) & P3(50) & $\mathrm{T} 6 \mathrm{~b}(90)$ & N5(100) & S3(80) & O1(70) & A1(90) & M2c(100) & 20.41 & Average \\
\hline 3 & $\mathrm{H} 4 \mathrm{~b}(90)$ & $\mathrm{D} 3 \mathrm{~b}(80)$ & P5(100) & $\mathrm{T} 5 \mathrm{~b}(80)$ & N5(100) & S5(25) & O1(70) & A1(90) & $\mathrm{M} 2 \mathrm{c}(100)$ & 9.072 & Poor \\
\hline 4 & $\mathrm{H} 4 \mathrm{~b}(90)$ & $\mathrm{D} 3 \mathrm{~b}(80)$ & P5(100) & Т6a(80) & N5(100) & $\mathrm{S} 4(40)$ & O1(70) & A2(95) & M2c(100) & 15.32 & Poor \\
\hline 5 & $\mathrm{H} 4 \mathrm{~b}(90)$ & $\mathrm{D} 3 \mathrm{~b}(80)$ & P5(100) & T6b(90) & N5(100) & S5(25) & O1(70) & A3(100) & $\mathrm{M} 2 \mathrm{c}(100)$ & 11.34 & Poor \\
\hline 6 & $\mathrm{H} 4 \mathrm{~b}(90)$ & $\mathrm{D} 3 \mathrm{~b}(80)$ & P5(100) & T6b(90) & N5(100) & S4(40) & $\mathrm{O} 1(70)$ & A3(100) & M2c(100) & 18.14 & Poor \\
\hline 7 & $\mathrm{H} 4 \mathrm{~b}(90)$ & $\mathrm{D} 3 \mathrm{~b}(80)$ & P5(100) & T6b(90) & N5(100) & S5(25) & O1(70) & A3(100) & $\mathrm{M} 2 \mathrm{c}(100)$ & 11.34 & Poor \\
\hline 8 & $\mathrm{H} 4 \mathrm{~b}(90)$ & $\mathrm{D} 3 \mathrm{~b}(80)$ & P5(100) & $\mathrm{T} 5 \mathrm{~b}(80)$ & N5(100) & S5(25) & $\mathrm{O} 1(70)$ & A1(90) & M2c(100) & 9.072 & Poor \\
\hline 9 & $\mathrm{H} 4 \mathrm{~b}(90)$ & $\mathrm{D} 3 \mathrm{~b}(80)$ & P5(100) & T6b(90) & N5(100) & $\mathrm{S} 4(40)$ & O1(70) & A2(95) & M2c(100) & 17.24 & Poor \\
\hline 10 & $\mathrm{H} 4 \mathrm{~b}(90)$ & $\mathrm{D} 3 \mathrm{~b}(80)$ & P5(100) & T6b(90) & N5(100) & $\mathrm{S} 5(25)$ & O1(70) & A3(100) & M2c(100) & 11.34 & Poor \\
\hline 11 & $\mathrm{H} 4 \mathrm{~b}(90)$ & $\mathrm{D} 3 \mathrm{~b}(80)$ & P5(100) & T6b(90) & N5(100) & S5(25) & O1(70) & A3(100) & M2c(100) & 11.34 & Poor \\
\hline
\end{tabular}

Table.3 Potential productivity index and Potential productivity class of Jammalamadugu -Prodduturtract of Pennar river basin in YSRK Adapa district

\begin{tabular}{|c|c|c|c|c|c|c|c|c|c|c|c|}
\hline Pedon No & $\begin{array}{c}\text { Moisture } \\
\text { (H) }\end{array}$ & $\begin{array}{l}\text { Drainage } \\
\text { (D) }\end{array}$ & $\begin{array}{l}\text { Effective } \\
\text { depth } \\
\text { (P) }\end{array}$ & $\begin{array}{l}\text { Texture/ } \\
\text { Structure } \\
\text { (T) }\end{array}$ & $\begin{array}{c}\text { Base } \\
\text { saturation } \\
(\mathrm{N})\end{array}$ & $\begin{array}{l}\text { Soluble } \\
\text { salts } \\
\text { (S) }\end{array}$ & $\begin{array}{c}\text { Organic } \\
\text { matter } \\
\text { content }(O)\end{array}$ & $\begin{array}{c}\text { Mineral } \\
\text { exchange } \\
\text { capacity (A) }\end{array}$ & $\begin{array}{l}\text { Mineral } \\
\text { reserve } \\
(\mathrm{M})\end{array}$ & $\begin{array}{c}\text { Potential } \\
\text { productivity } \\
\text { index }\end{array}$ & $\begin{array}{c}\text { Potential } \\
\text { productivity } \\
\text { class }\end{array}$ \\
\hline 1 & $\mathrm{H} 4 \mathrm{~b}(90)$ & D4(100) & P3(50) & T6b(90) & N5(100) & S1(100) & O4(100) & A2(95) & M2c(100) & 38.46 & Good \\
\hline 2 & $\mathrm{H} 4 \mathrm{~b}(90)$ & D4(100) & P3(50) & T6b(90) & N5(100) & $\mathrm{S} 1(100)$ & $\mathrm{O} 4(100)$ & A1(90) & M2c(100) & 36.45 & Good \\
\hline 3 & $\mathrm{H} 4 \mathrm{~b}(90)$ & $\mathrm{D} 3 \mathrm{~b}(80)$ & P5(100) & $\mathrm{T} 5 \mathrm{~b}(80)$ & N5(100) & S1(100) & O2(80) & A1(90) & M2c(100) & 41.47 & Good \\
\hline 4 & $\mathrm{H} 4 \mathrm{~b}(90)$ & $\mathrm{D} 3 \mathrm{~b}(80)$ & P5(100) & Т6а(80) & N5(100) & S1(100) & $\mathrm{O} 2(80)$ & A2(95) & M2c(100) & 43.77 & Good \\
\hline 5 & $\mathrm{H} 4 \mathrm{~b}(90)$ & $\mathrm{D} 3 \mathrm{~b}(80)$ & P5(100) & T6b(90) & N5(100) & S1(100) & $\mathrm{O} 2(80)$ & A3(100) & M2c(100) & 51.84 & Good \\
\hline 6 & $\mathrm{H} 4 \mathrm{~b}(90)$ & $\mathrm{D} 3 \mathrm{~b}(80)$ & P5(100) & $\mathrm{T} 6 \mathrm{~b}(90)$ & N5(100) & S1(100) & $\mathrm{O} 2(80)$ & A3(100) & M2c(100) & 51.84 & Good \\
\hline 7 & $\mathrm{H} 4 \mathrm{~b}(90)$ & $\mathrm{D} 3 \mathrm{~b}(80)$ & P5(100) & $\mathrm{T} 6 \mathrm{~b}(90)$ & N5(100) & $\mathrm{S} 1(100)$ & $\mathrm{O} 2(80)$ & $\mathrm{A} 3(100)$ & M2c(100) & 51.84 & Good \\
\hline 8 & $\mathrm{H} 4 \mathrm{~b}(90)$ & $\mathrm{D} 3 \mathrm{~b}(80)$ & P5(100) & $\mathrm{T} 5 \mathrm{~b}(80)$ & N5(100) & S1(100) & $\mathrm{O} 2(80)$ & A1(90) & M2c(100) & 41.47 & Good \\
\hline 9 & $\mathrm{H} 4 \mathrm{~b}(90)$ & $\mathrm{D} 3 \mathrm{~b}(80)$ & P5(100) & $\mathrm{T} 6 \mathrm{~b}(90)$ & N5(100) & S1(100) & $\mathrm{O} 2(80)$ & A2(95) & M2c(100) & 49.25 & Good \\
\hline 10 & $\mathrm{H} 4 \mathrm{~b}(90)$ & $\mathrm{D} 3 \mathrm{~b}(80)$ & P5(100) & T6b(90) & N5(100) & S1(100) & $\mathrm{O} 2(80)$ & A3(100) & M2c(100) & 51.84 & Good \\
\hline 11 & $\mathrm{H} 4 \mathrm{~b}(90)$ & $\mathrm{D} 3 \mathrm{~b}(80)$ & P5(100) & T6b(90) & N5(100) & S1(100) & $\mathrm{O} 2(80)$ & A3(100) & M2c(100) & 51.84 & Good \\
\hline
\end{tabular}


Table.4 Soil productivity index and capability classes

\begin{tabular}{|c|c|c|c|c|c|}
\hline \multirow{2}{*}{ Pedon No } & \multicolumn{2}{|c|}{$\begin{array}{c}\text { Actual } \\
\text { productivity(P) }\end{array}$} & \multicolumn{2}{c|}{$\begin{array}{c}\text { Potential } \\
\text { Productivity(P') }\end{array}$} & $\begin{array}{c}\text { Crop improvement } \\
\text { factor(Ci) }\end{array}$ \\
\cline { 2 - 5 } & Rating & Classes & Rating & Classes & \\
\hline $\mathbf{1}$ & 21.54 & Average & 38.46 & Good & 1.78 \\
\hline $\mathbf{2}$ & 20.41 & Average & 36.45 & Good & 1.78 \\
\hline $\mathbf{3}$ & 9.072 & Poor & 41.47 & Good & 4.57 \\
\hline $\mathbf{4}$ & 15.32 & Poor & 43.77 & Good & 2.86 \\
\hline $\mathbf{5}$ & 11.34 & Poor & 51.84 & Good & 4.57 \\
\hline $\mathbf{6}$ & 18.14 & Poor & 51.84 & Good & 2.86 \\
\hline $\mathbf{7}$ & 11.34 & Poor & 51.84 & Good & 4.57 \\
\hline $\mathbf{8}$ & 9.072 & Poor & 41.47 & Good & 4.57 \\
\hline $\mathbf{9}$ & 17.24 & Poor & 49.25 & Good & 2.86 \\
\hline $\mathbf{1 0}$ & 11.34 & Poor & 51.84 & Good & 4.57 \\
\hline $\mathbf{1 1}$ & 11.34 & Poor & 51.84 & Good & 4.57 \\
\hline
\end{tabular}

Fig.1 Landscape diagrams of Gandikota formation in Jammalamadugu - Proddutur tract of Pennarriver basin in YSR Kadapa district
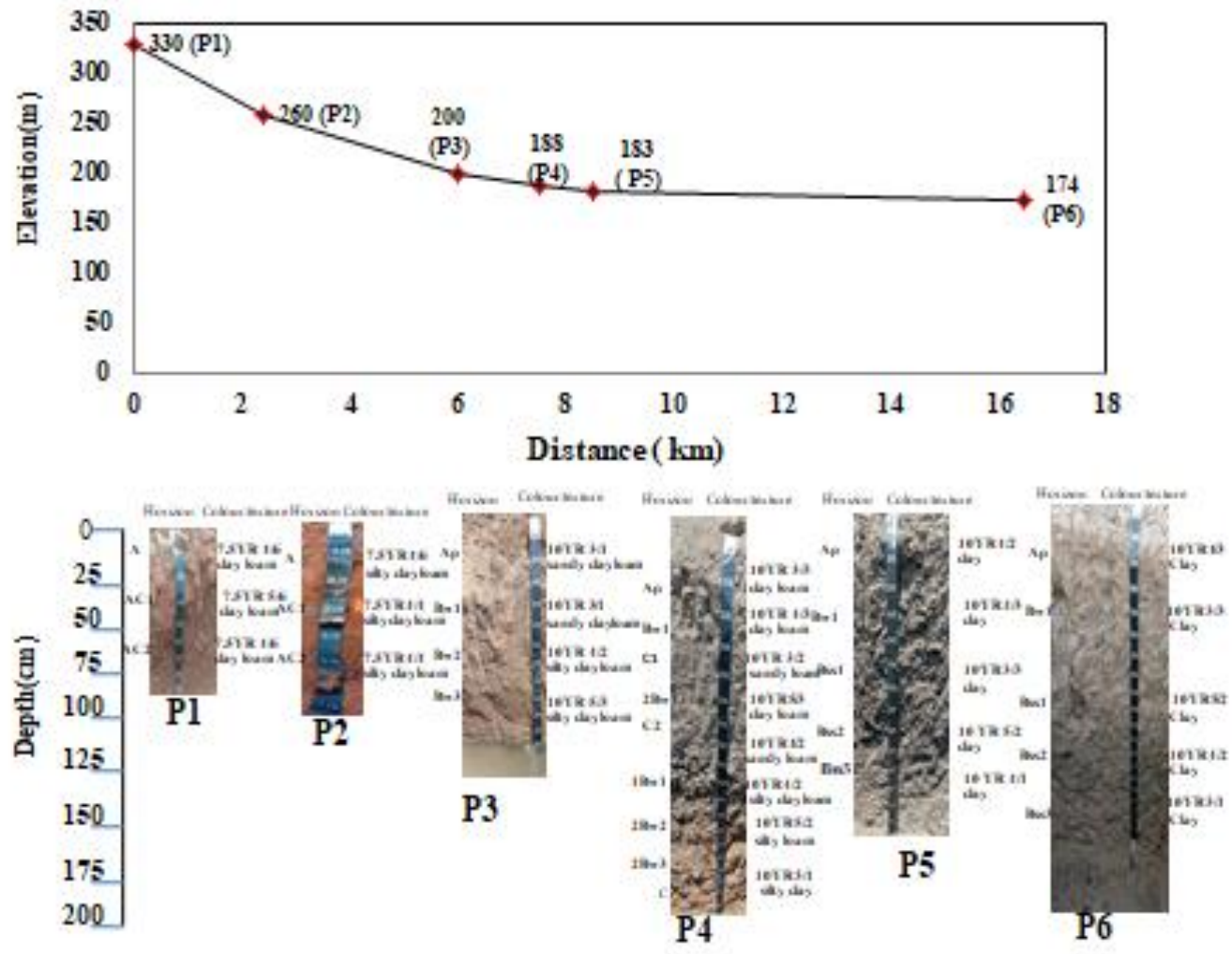
Fig.2 Land scape diagrams of Tadipatriformation in Jammalamadugu - Proddutur tract of Pennar river basin in YSR Kadapa district

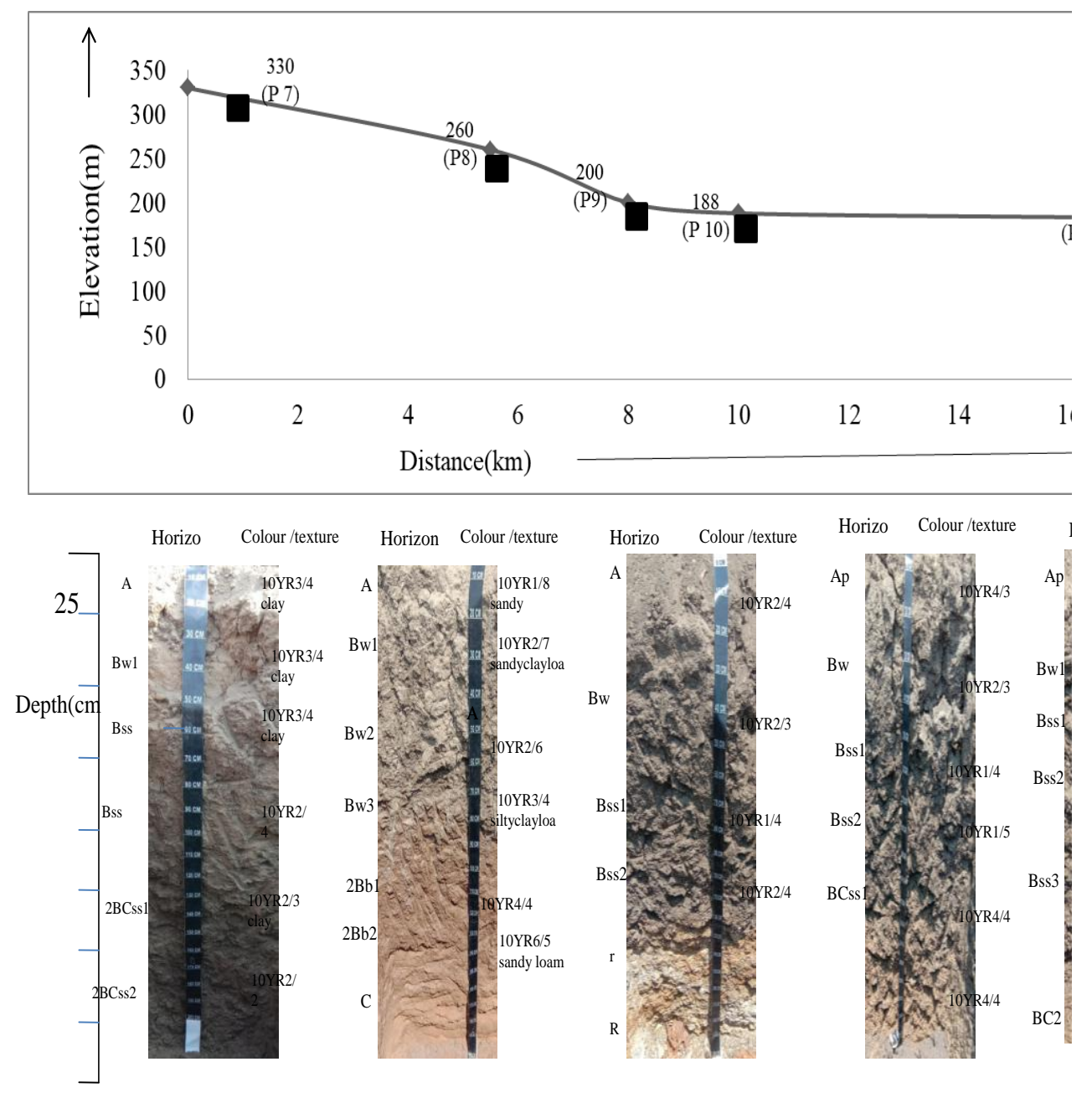

The actual productivity of the studied soils/ pedons varied from 9.07 to 21.54 whereas potential productivity varied between 36.45 and 51.84 (Tables 2 and 3). The lowest potential productivity was observed in pedon 2 (36.45) while the highest potential productivity was observed in pedons6, 7, 10 and 11 (51.84). In general the potential productivity of most of the pedons was categorized under the good productivity class. Quantitatively, the coefficient of improvement in the present study ranged from 1.78 to 4.57. Based on the Riquier's parametric approach, the soils of
Jammalamadugu - Proddutur tract of Pennar river basin in YSR Kadapa district had one category i.e. good (Table 4).

The actual productivity of pedons 1 (21.54) and 2 (20.41) was average whereas the actual productivity of pedons 3 to 11 was poor. The main constraints attributed for average productivity of pedons 1 and 2 are low organic carbon, slope, shallow depth and erosion. The low organic carbon can be improved by application of FYM /compost, adopting green manuring and incorporation of crop residues (Shireesha et al., 2020). 
Furthermore, practice of contour farming or contour bunding to restrict the erosion hazards and deepening the soil by ridging and deep ploughing in shallow soils can be taken up. Therefore, it is also suggested to take up alternate crops like vegetables, pulses, oil seeds and ground nut crops which suits better for shallow soils with loamy sand structure in pedons having poor or average productivity (Gangopadhyay et al., 2012). By adopting above management practices the potential productivity of the pedons 1 and 2 can be upgraded from average to good.

Similarly, the actual productivity of pedons 3 to 11was poor. The main constraints attributed for poor productivity of pedons 3 to 11 are slope, low organic carbon, shallow depth, erosion, drainage and texture. The low organic carbon, erosion and shallow depth improved by adopting the above management practices. However, texture and drainage in pedons 3 to 11 can be improved by mixing of soil with tank (Girish et al., 2010.) Due to adoption of above management practices the potential productivity of the pedons 3 to 11 can be upgraded from poor to good.

In conclusion, the actual productivity can be upgraded from average to good (P1 and P2) and poor to good (P3 to P11) by adopting improved management practices such as application of compost/ FYM or adopting green manuring, incorporation of crop residues, trash mulching and vertical mulching with filter cake, mixing of soil with tank silt and contour bunding and contour farming.

\section{References}

AIS\&LUS. 1970 Soil survey manual, All India soil and land use survey organization, IARI, New Delhi, pp. 163.

Bower, C.A., Reitemeier, R.F and Fireman,
M. 1952.Exchangeable cations analysis of saline and alkali soils. Soil science. 73: 251-261.

Gangopadhyay, S.K., DipakSarkar, Sahoo, A.K and Singh, S.K. 2012.Soils of the rainfed region of West Bengal and their Productivity potential appraisal. Journal of the Indian society of soil science. 60 (2): 83-91.

Girish, B.P., Nagaraju, M.S.S., Prasad, J and Srivastava, R. 2010. Characterization, evaluation and mapping of land resources in Lendi watershed of Chandrapur district of Maharashtra using remote sensing and GIS. Journal of the Indian society of soil science. 58(4): 442-448.

Jackson, M.L.1973. Soil chemical analysis. Oxford IBH publishing house, Bombay. 38.

Niranjana, K.V., Ramamurthy, V., Rajendra Hegde, Srinivas, S., ArtiKoyal, Naidu, L.G.K and Sarkar, D. 2011. Characterization, classification and suitability evaluation of banana growing soils of Pulivendula region of Andhra Pradesh. Journal of the Indian Society of Soil Science. 59: 1-5.

Piper, C.S. 1966. Soil and plant analysis. Hans's publications, Bombay. pp. 59.

Riquier, J., Bramo, D.L and Cornet, J.P. 1970. A new system of soil appraisal in terms of actual and potential productivity.FAO, soil resource development and conservation service, land and water development division, FAO, Rome.Pp.88-99.

Sharma, P.D. 2006. Soil science research vision. 2025. Indian society of soil science newsletter. 20, p.1.

Sireesha, P.V.G and Naidu, M.V.S. 2020. Production potential appraisal: a case study in Banaganapalle mandal of Kurnool district in Andhra Pradesh, India.

Soil Survey Division Staff. 2000. Soil survey 
manual (Indian print), USDA hand book No.18, US govt. printing office, Washington, DC.

Soil Survey Staff. (2014). Keys to soil taxonomy (Twelfth edition), USDA, Natural resource conservation service, Washington, DC.

\section{How to cite this article:}

Sujatha, D. V., M. V. S. Naidu, B. P. Bhaskar, D. Subramanyam and Ravindra Reddy, B. 2020. Production Potential Appraisal: A Case Study in Jammalamadugu- Proddutur Tract of Pennar River Basin in YSR Kadapa District, Andhra Pradesh, India. Int.J.Curr.Microbiol.App.Sci. 9(10): 1516-1523. doi: https://doi.org/10.20546/ijcmas.2020.910.181 